\title{
37. Maternal Exposure of Rats to Sodium Valproate Develops Tolerance to Valproate Itself in the Offspring*)
}

\author{
By Tomoko FuJII, Naomi Hara, and Yuuko Kuga \\ Department of Pharmacology, Teikyo University \\ School of Medicine, Tokyo 173 \\ (Communicated by Setsuro Ebashi, M. J. A., April 14, 1986)
}

There is accumulating evidence showing that maternal exposure of rats or mice to central nervous system(CNS)-acting drugs induces latent and/or longlasting abnormalities in the brain function of the offspring without any morphological alterations. This could be detected by evaluating pharmacological responses of the animals to acute administration of several drugs. We have previously reported that maternal exposure of the rat to phenytoin, an antiepileptic drug, produced a prolongation of cataleptic responses to haloperidol in the offspring as assessed in adulthood.1) Maternal exposure to phenobarbital, a well known anticonvulsant drug, produced alterations in the pentobarbitalinduced sleeping time ${ }^{2}$ ) and in the thermic response to chlorpromazine. ${ }^{3)}$

In the present experiment, the effect of prenatal exposure of rats to sodium valproate, another major anticonvulsant drug, was studied and a significant reduction in the anticonvulsive effect of valproate was found in the exposed rats. Wistar-Imamichi rats were mated and the day on which sperm was present in the vaginal smear was designated as day 0 of pregnancy. Pregnant rats were injected s.c. once daily with $1,5,10$ or $20 \mathrm{mg} / \mathrm{kg}$ of sodium valproate (Kyowa Hakko Kogyo Co.) from days 5-21 of gestation. The drug was dissolved in distilled water to give an injection volume of $1 \mathrm{ml} / \mathrm{kg}$. Control pregnant rats received saline. The animals were kept in a temperature controlled room $\left(22 \pm 3^{\circ} \mathrm{C}\right)$ with a lighting schedule for $14 \mathrm{hr}$ light (0600-2000) and $10 \mathrm{hr}$ darkness and supplied a stock diet (Oriental Yeast Co.) and water ad libitum. Pentobarbitalinduced sleeping time in the $F_{1}$ rats was measured at 5 weeks of age; $30 \mathrm{mg} / \mathrm{kg}$ of sodium pentobarbital (Nembutal Injection ${ }^{\circledR}$, Dainippon Pharmaceutical Co.) was injected i.p. and the duration of the loss of righting reflex was measured. Anticonvulsive $\mathrm{ED}_{\tilde{\jmath} 0}$ values for sodium valproate(VP) against pentylenetetrazolinduced convulsions were estimated for 7 -week-old control- $\mathrm{F}_{1}$ and VP-F $\mathrm{F}_{1}$ rats of each maternal dose group using the "Up and Down" method.4) Two to three determinations were performed for each dose group. Sodium valproate was injected i.p. to the rats $15 \mathrm{~min}$ before the $\mathrm{PTZ}(120 \mathrm{mg} / \mathrm{kg}$, s.c.) administration and the dose protecting the death with clonic-tonic convulsive seizures within $30 \mathrm{~min}$ after PTZ administration was estimated. Minimal seizure threshold in male and female $F_{1}$ rats of the control and VP groups at 7 weeks of age was estimated as $\mathrm{ED}_{\bar{\jmath} 0}$ of PTZ by the "Up and Down" method; the threshold dose of PTZ(s.c.) to induce clonic convulsions within $15 \mathrm{~min}$ was calculated. The $\mathrm{LD}_{\overline{5} 0}$ of PTZ in 7-week-old control male and female rats was estimated by the

*) We wish to thank The Kyowa Hakko Kogyo Ltd. for his generous gift of valproate. This work was supported in part by a grant from The Shimabara Science Promotion Foundation, Japan. 
"Up and Down" method; death within 30 min with clonic-tonic convulsions was recorded.

Results and discussion. There were no significant differences in the littersize and growth rate of the VP- $\mathrm{F}_{1}$ rats as compared with those in control- $\mathrm{F}_{1}$ rats. The time of the eye-opening and of vaginal opening was normal. Pentobarbital-induced sleeping time in the $V A-F_{1}$ rats was comparable to that in controls. A marked sex difference in the anticonvulsive $\mathrm{ED}_{\tilde{5} 0}$ values for valproate against PTZ-induced convulsions was found in both control and VP-F $F_{1}$ rats; the values in female rats were significantly lower than in males (Fig. 1). Further-

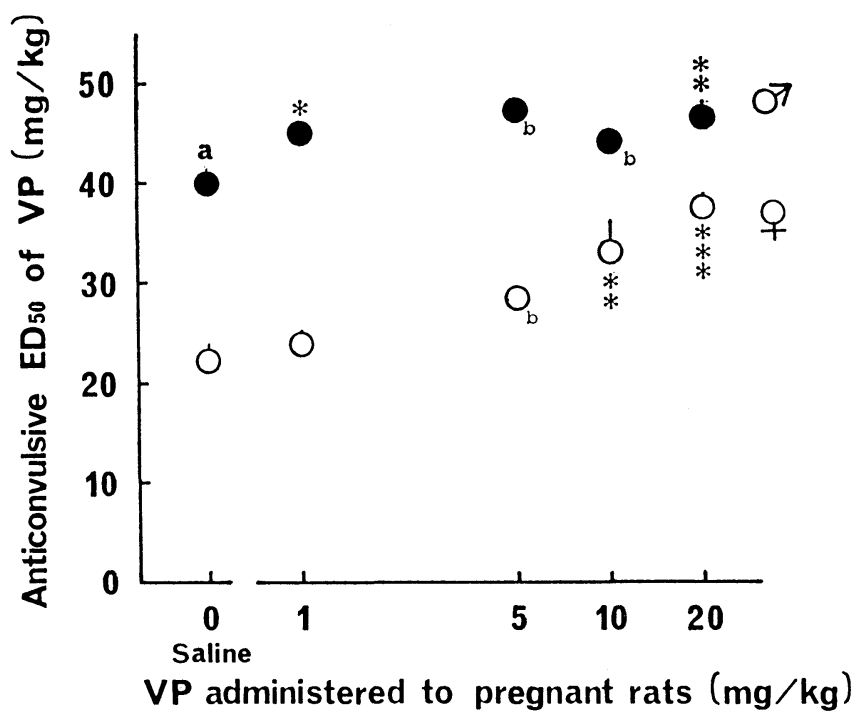

Fig. 1. Anticonvulsive $E D_{50}$ values for valproate (VP) against pentylenetetrazolinduced convulsions in 7-week-old rats exposed to valproate from days 5-21 of gestation. a, $\mathrm{P}<0.01$ vs. female; $* \mathrm{P}<0.05$; $* * \mathrm{P}<0.01$; ** $\mathrm{P}<0.001$ vs. the value of 0 -group; $b$, the average of two determinations.

more, the anticonvulsive $\mathrm{ED}_{50}$ values in both male and female of the VP-F $\mathrm{F}_{1}$ rats were higher than those in control- $\mathrm{F}_{1}$ rats. The $\mathrm{ED}_{50}$ values for valproate in the VP- $F_{1}$ female rats increased progressively parallel with the dose of valproate given to their mothers (Fig. 1), whereas in the VP-F 1 male rats, the $\mathrm{ED}_{50}$ values for valproate reached a plateau at the dose of $5 \mathrm{mg} / \mathrm{kg}$ given to mothers. There was no significant difference in the minimal seizure threshold against PTZ between sexes and between control- $F_{1}$ and VP-F 1 groups, and in the $\mathrm{LD}_{50}$ values of PTZ between sexes in control rats (Table I).

The present results indicate that rats exposed to valproate during their fetal life develops tolerance to valproate. Tolerance to drugs can be produced mainly by (1) metabolic changes of drugs in the liver and other tissues, or by (2) a reduction in the normal reactivity of the cells at the target site of drugs. The VP- $F_{1}$ rats in the present experiment did not show any significant changes in the minimal seizure threshold to PTZ as compared with that in the control-F $F_{1}$ rats. Major anticonvulsant drugs, phenytoin and carbamazepine, have been known to induce a reduction in the effect during chronic treatment. However, the $F_{1}$ 
[Vol. $62(\mathrm{~B})$,

rats of carbamazepine-treated mothers did not show any increase in the anticonvulsive dose of carbamazepine against PTZ-induced clonic-tonic convulsions (Fujii, unpublished results), suggesting that the mechanism involved in the development of tolerance to valproate in the $\mathrm{VP}-\mathrm{F}_{1}$ rats may be specifically related to the molecular structure of valproate and to the valproate target site in the

Table I. Convulsive $\mathrm{ED}_{50}$ and $\mathrm{LD}_{50}$ values for pentylenetetrazol (s. c.) in 7-week-old $\mathrm{F}_{1}$ rats

\begin{tabular}{clllll}
\hline \multirow{2}{*}{ Group } & \multicolumn{2}{c}{$\mathrm{ED}_{50}(\mathrm{mg} / \mathrm{kg})$} & & \multicolumn{2}{c}{$\mathrm{LD}_{50}(\mathrm{mg} / \mathrm{kg})$} \\
\cline { 2 - 3 } \cline { 5 - 6 } & \multicolumn{1}{c}{ Male } & Female & & Male & Female \\
Saline & $51.41 \pm 2.46^{*}$ & $56.41 \pm 1.01$ & & $111.6 \pm 6.0^{*}$ & $113.3 \pm 3.9$ \\
VP 10 & $50.11^{* *}$ & $56.03^{* *}$ & & & \\
\hline
\end{tabular}

* Mean \pm S.E.M. of 3 determinations by the "Up and Down" method. ** The average of 2 determinations.

central nervous system. Mother rats treated with $10 \mathrm{mg} / \mathrm{kg}$ valproate were tested for the possibility of the development of tolerance to valproate two weeks after weaning. Anticonvulsive $\mathrm{ED}_{\bar{\jmath} 0}$ values for valproate against PTZ-induced convulsions was $19.05 \mathrm{mg} / \mathrm{kg}$ for the VP-treated mothers and $16.41 \mathrm{mg} / \mathrm{kg}$ for the saline-treated mothers when estimated by the "Up and Down" method (the dose of PTZ injected was $150 \mathrm{mg} / \mathrm{kg}$ instead of $120 \mathrm{mg} / \mathrm{kg}$ ). The value in the VPtreated mothers was thus only slightly higher than that in the saline-treated mothers, though only one estimation was available using 10 rats for each group. In contrast, the $\mathrm{ED}_{50}$ value in the $10 \mathrm{mg} / \mathrm{kg}$ VP- $\mathrm{F}_{1}$ female rats was much higher than that in the control- $F_{1}$ rats as shown in Fig. 1. Together with these results, the finding of the sex-related difference in the maternal-dose dependent increase in the magnitude of valproate tolerance in the $\mathrm{VP}-\mathrm{F}_{1}$ rats may suggest that the cause of the tolerance to valproate could be due to changes in the sensitivity of the central nervous system to valproate itself. Valproic acid is a useful antiepileptic drug which is effective against a wide spectrum of epileptic seizures, particularly of the petit mal type, though the mechanism of its action is not completely understood. Valproate has been observed to increase the brain content of $\gamma$-aminobutyric acid(GABA) ${ }^{5}$ ) and this is generally considered to be the basis of its anticonvulsive effect. However, the suppression of seizures by valproate does not always appear to be mediated by cerebral GABA, $\left.{ }^{6}\right)$ indicating that other neurotransmitters may also be involved. It has recently been suggested that spinal 5-hydroxytryptamine may play a role in reduction of clonic PTZ convulsions following treatment with valproic acid. ${ }^{7}$ ) The mechanism of anticonvulsive action of valproate differs from that of phenytoin and carbamazepine. Phenytoin and carbamazepine can displace benzodiazepine binding sites. Binding to these "anticonvulsant" sites regulates $\mathrm{Ca}^{2+}$-calmodulin-stimulated membrane protein phosphorylation and depolarization-dependent $\mathrm{Ca}^{2+}$ uptake in intact synaptosome preparation. ${ }^{8)}$ However, valproate does not have major effects on synaptic phosphorylation or on neurotransmitter release. ${ }^{8)}$ Altered neurotransmitter function or altered neuronal membrane properties could be involved as factors producing the tolerance to valproate. Major antiepileptic drugs, phenytoin, ${ }^{9)}$ phenobarbital ${ }^{10}$ ) and carbamazepine ${ }^{11}$ ) have been observed in humans to pass the placenta from 
maternal site to fetal circulation very rapidly. Chronic administration of valproate has been found to result in an increase in the brain concentration of valproate metabolites despite its decrease in the liver. ${ }^{12)}$ Continued exposure to a rather high level of valproate or its biologically active metabolites during fetal life would result in the abnormal maturation of the target sites of valproate in the central nervous system.

Recently, sexual dimorphism in the structure and function of the central nervous system has received considerable attention. We have reported several results showing sex-related alterations in the response to various drugs in the offspring of rats exposed to drugs during pregnancy. Maternal exposure of rats to phenytoin resulted in the development of hypersensitivity to haloperidol at a greater extent in the female offspring. ${ }^{1)}$ Maternal exposure to diazepam induced a hypersensitive response to injected thyrotropin-releasing hormone only in female offspring in adulthood. ${ }^{13)}$ Prenatal exposure to imipramine affected male offspring to a greater extent: The hyperthermic response to chlorpromazine was shown, while the littermate females and control male and female rats showed a marked hypothermia. ${ }^{14)}$ The problem on the possible involvement of sex steroids in the functional maturation of the fetal brain of animals remains to be solved.

\section{References}

1) Fujii, T., Ikeda, H., and Yamamoto, N.: Proc. Japan Acad., 58B, 78-82 (1982).

2) Fujii, T. et al.: Antiepileptic Drugs and Pregnancy (eds. T. Sato and S. Shinagawa). Excerpta Medica, Amsterdam-Princeton-Geneva-Tokyo, 219-232 (1984).

3) Fujii, T. et al.: Neuropharmacology (1986) (in press).

4) Dixon, W. J.: J. Amer. Statist. Ass., 60, 967-978 (1965).

5) Godin, Y. et al.: J. Neurochem., 16, 869-873 (1969).

6) Goldstein, D. B.: J. Pharmacol. Exp. Therap., 208, 223-227 (1979).

7) Lazarova, M., Bendotti, C., and Samanin, R.: Naunyn-Schmiedeberg's Arch. Pharmacol., 322, 147-152 (1983).

8) De Lorenzo, R. J.: Ann. Neurol., 16, suppl., S104-114 (1984).

9) Westmoreland, B., and Bass, N. H.: Arch. Neurol., 24, 158-164 (1971).

10) Melchior, J. C. et al.: Lancet, 2, 860-861 (1967).

11) Hosokawa, K. et al.: Igaku No Ayumi, 105, 941-943 (1978).

12) Carl, G. F., DeLoach, C., and Patterson, J.: Life Sci., 37, 2029-2035 (1985).

13) Fujii, T., Yamamoto, N., and Fuchino, K.: Toxicol. Lett., 16, 131-137 (1983).

14) Fujii, T., and Ohtaki, Y.: Develop. Pharmacol. Therap., 8, 364-373 (1985). 